\title{
On New Families of Integrals in Analytical Studies of Superconductors within the Conformal Transformation Method
}

\author{
Ryszard Gonczarek, ${ }^{1}$ Mateusz Krzyzosiak, ${ }^{2}$ Adam Gonczarek, ${ }^{3}$ and Lucjan Jacak ${ }^{1}$ \\ ${ }^{1}$ Faculty of Fundamental Problems of Technology, Wrocław University of Technology, Wybrzeże Wyspiańskiego 27, \\ 50-370 Wrocław, Poland \\ ${ }^{2}$ University of Michigan and Shanghai Jiao Tong University Joint Institute, 800 Dongchuan Road, Shanghai 200240, China \\ ${ }^{3}$ Faculty of Computer Science and Management, Wrocław University of Technology, Wybrzeże Wyspiańskiego 27, \\ 50-370 Wrocław, Poland \\ Correspondence should be addressed to Ryszard Gonczarek; ryszard.gonczarek@pwr.edu.pl
}

Received 19 April 2015; Accepted 2 July 2015

Academic Editor: Jaume Gázquez

Copyright (C) 2015 Ryszard Gonczarek et al. This is an open access article distributed under the Creative Commons Attribution License, which permits unrestricted use, distribution, and reproduction in any medium, provided the original work is properly cited.

\begin{abstract}
We show that, by applying the conformal transformation method, strongly correlated superconducting systems can be discussed in terms of the Fermi liquid with a variable density of states function. Within this approach, it is possible to formulate and carry out purely analytical study based on a set of fundamental equations. After presenting the mathematical structure of the $s$ wave superconducting gap and other quantitative characteristics of superconductors, we evaluate and discuss integrals inherent in fundamental equations describing superconducting systems. The results presented here extend the approach formulated by Abrikosov and Maki, which was restricted to the first-order expansion. A few infinite families of integrals are derived and allow us to express the fundamental equations by means of analytical formulas. They can be then exploited in order to find quantitative characteristics of superconducting systems by the method of successive approximations. We show that the results can be applied in studies of high- $T_{c}$ superconductors and other superconducting materials of the new generation.
\end{abstract}

\section{Introduction}

Over the past few years, research on superconductivity has been gaining new momentum due to the milestone discovery of iron-based superconductors $[1,2]$. Along with the ongoing work on high- $T_{c}$ materials, studies on superconducting systems of the new generation remain one of the most active research areas in superconductivity research. Progress in fabrication and characterization techniques is mutually coupled with efforts to model and predict theoretically the trends and phenomena observed in experiments. An important tool for quantitative theoretical studies on superconducting systems is the gap equation accompanied by the carrier concentration equation. It appears in similar forms in the BCS theory, the Eliashberg equations, the Van Hove Scenario, and the conformal transformation method, with the latter taking fully into account the low-dimensional structure of high- $T_{c}$ materials. In order to evaluate fundamental thermodynamic functions such as the free energy, the thermodynamic potential, the entropy, the heat capacity, and other quantitative parameters such as the transition temperature, the heat capacity leap, the critical current, the critical magnetic field, and the isotope shift, one should study the gap equation and the carrier concentration equation along with the corresponding formulas for the thermodynamic potential, the critical fields, the concentration of superconducting carriers, and so forth [3-11].

Employing the gap equation with a fixed chemical potential equal to the Fermi energy, in theoretical studies of BCStype superconducting systems at subcritical temperatures, Abrikosov and Maki have suggested to apply an expansion in the integral equation and restrict it to the first-order terms. The calculated integral allowed them to estimate the solution of the first boundary value problem for the gap of a BCS superconductor as a function of the temperature [12-14].

Contemporary studies focus, however, more on superconducting materials of the new generation. These include 
high- $T_{c}$ copper-oxide quasi two-dimensional superconducting systems and various doped superconducting compounds such as spinel- and perovskite-type structures of superconducting compounds of a trivalent rare-earth and a divalent alkali-earth ion. Other classes of superconducting materials that have been actively studied in recent years include novel superconducting compounds of $\mathrm{MgB}_{2}$ with a $\mathrm{C}, \mathrm{Al}$, or $\mathrm{Sc}$ substitution, or organic superconductors with a controlled bandwidth and band filling [7, 11, 15-22]. Theoretical description of such superconducting systems requires to take into account spin-fluctuations or strong-correlation effects. These can be included by means of an effective Hamiltonian in the strongly interacting Hubbard model with a given (multiband) one-particle dispersion relation enriched by selfenergy corrections, as well as a quite general form of the pairing potential. The latter can be decomposed into an antisymmetric and a symmetric part determining the symmetry of the order parameter [23-30].

In order to address the above problems in a systematic analytical manner, we propose to apply the conformal transformation, which allows us to consider a superconducting system as mapped onto a Fermi liquid in an isotropic momentum space with a naturally introduced function of the density of states [23]. In further discussion, a series expansion of fundamental integrals emerging in the formalism proves to be a powerful tool. In the present paper, we derive formulas for some infinite families of integrals, which allow us to construct such series up to an arbitrary order. We emphasize that until now only few of such integrals were given [12-14, 31-33].

\section{Employed Formalism}

Employing the Green function formalism, with the effects of an external magnetic field included, one can derive a set of two fundamental equations in the momentum space, consistent with the mean-field approximation. One of these equations is the gap equation $[5,7,19-21,32,34]$ :

$$
\begin{aligned}
\Delta_{\mathbf{k}} & =\sum_{\mathbf{k}^{\prime}} V\left(\mathbf{k}, \mathbf{k}^{\prime}\right) \frac{\Delta_{\mathbf{k}^{\prime}}}{2 E_{\mathbf{k}^{\prime}}}\left(\tanh \frac{E_{\mathbf{k}^{\prime}}+m \mathbf{v}\left(\partial \epsilon_{\mathbf{k}^{\prime}} / \partial \mathbf{k}^{\prime}\right)}{2 T}\right. \\
& \left.+\tanh \frac{E_{\mathbf{k}^{\prime}}-m \mathbf{v}\left(\partial \epsilon_{\mathbf{k}^{\prime}} / \partial \mathbf{k}^{\prime}\right)}{2 T}\right),
\end{aligned}
$$

where $E_{\mathbf{k}}=\sqrt{\left(\epsilon_{\mathbf{k}}-\mu\right)^{2}+\Delta_{\mathbf{k}}^{2}}$ and $\mathbf{v}=\nabla \varphi-(e / m) \mathbf{A}$ is the superflow, with $\varphi$ being the phase of the order parameter and $\mathbf{A}$ is the vector potential. The other one is the carrier concentration equation:

$$
\begin{aligned}
n= & \frac{1}{N} \sum_{\mathbf{k}}\left[1-\frac{\epsilon_{\mathbf{k}}-\mu}{2 E_{\mathbf{k}}}\left(\tanh \frac{E_{\mathbf{k}}+m \mathbf{v}\left(\partial \epsilon_{\mathbf{k}} / \partial \mathbf{k}\right)}{2 T}\right.\right. \\
& \left.\left.+\tanh \frac{E_{\mathbf{k}}-m \mathbf{v}\left(\partial \epsilon_{\mathbf{k}} / \partial \mathbf{k}\right)}{2 T}\right)\right],
\end{aligned}
$$

which determines the chemical potential $\mu$. This equation allows one to express $\mu$ in (1) in dependence on $n$ and study some superconducting systems with a partially filled conduction band. The dispersion relation entering both equations is usually defined with respect to the Fermi level.

In the BCS theory, which was greatly successful in explaining various properties of simple low- $T_{c}$ superconductors by means of several experimental parameters, an electron system is treated as a Fermi liquid. However, high$T_{c}$ materials are complicated, and many fundamental condensed matter physics phenomena play some role in one or another part of their phase diagram. The normal state near the composition corresponding to the highest $T_{c}$ shows a convincing evidence of being a more composed form of a quantum liquid than the Fermi liquid and should be considered as a non-Fermi liquid. In a wide class of nonFermi liquids presented in [35] as applicable to modeling of superconducting systems, for some of them, a singular (or marginal) Fermi-liquid behavior is observed in the underdoped (low carrier concentration) regime, whereas the other ones exhibit it in the overdoped (high carrier concentration) regime, near to the optimally doped structure. Therefore, low$T_{c}$ superconductors and overdoped high- $T_{c}$ cuprate superconductors, described by an effective Hamiltonian with a nonlinear dispersion relation $\epsilon_{\mathbf{k}}$, can be discussed in terms of the Fermi liquid after applying a conformal transformation of the momentum space.

In general, such an approach can be applied to anisotropic superconducting systems with an arbitrary dispersion relation for spin-singlet $s$-wave or $d$-wave symmetry states, as well as for the spin-triplet $p$-wave symmetry state, with a fixed carrier concentration. It also allows one to include the effects induced by variations of the carrier concentration $n$, and the pairing potential amplitudes in both the isotropic and the anisotropic channel, in order to discuss the stability of these symmetry states $[5,20,27,33-36]$.

Studies of the pairing potential seem to be particularly interesting and valuable, since a definite pairing mechanism in high- $T_{c}$ superconducting systems has not been clearly identified yet. However, in the literature, two mechanisms are usually discussed [26]. The first one is based on the singleband Hubbard model (or related models, such as the $t$ - $J$ model) whereas in the other one it is suggested that the role of the electron-phonon interaction is crucial. Quantitative analysis of the pairing interaction in superconducting systems could therefore provide useful insights into the mechanism of Cooper pairs formation in superconducting systems of the new generation $[7,33]$. In our approach, we have introduced the pairing potential amplitude in the form $V\left(\mathbf{k}, \mathbf{k}^{\prime}\right)$. Nevertheless, one should remember that $-V\left(\mathbf{k}, \mathbf{k}^{\prime}\right)$ is the momentum-dependent part of the full pairing potential, which is an antisymmetric matrix-function defined in the momentum-spin space. This function is obtained as a result of the Fourier transform of an effective two-particle interaction potential including all many-body effects, and ensuring the spin conservation law [36]. Hence, $-V\left(\mathbf{k}, \mathbf{k}^{\prime}\right)$ is a symmetric function of $\mathbf{k}$ and $\mathbf{k}^{\prime}$, and in order to generate attraction needed to form a paired state in the system, this function must be negative. In the formulated approaches (cf. $[4,5,14$, $26])$, the function $-V\left(\mathbf{k}, \mathbf{k}^{\prime}\right)$ becomes negative and represents the introduced pairing potential amplitude $V\left(\mathbf{k}, \mathbf{k}^{\prime}\right)$ only in 
a small region of the momentum space near to the Fermi surface, and so (for a spin-singlet $s$-wave state) it can be consider as constant.

2.1. Integrals of the Fundamental Equations for $\mathbf{v}=0$. It is worth to emphasize once again that the conformal transformation method $[7,23,37]$ proves that an arbitrary anisotropic system, with a given one-particle energy spectrum, can be considered as an isotropic Fermi liquid in the momentum space. However, it needs then to be taken into account that quantum-mechanical states are not distributed homogeneously. The distribution is defined by a scalar field of the density of states, which for a fixed spin-singlet $s$-wave state, with a given carrier concentration $n$ and a pairing potential amplitude $V_{0}$, reduces to the density of states function. Therefore, by applying the conformal transformation method to an anisotropic superconductor with a given dispersion relation, one can map quasiparticle excitations onto Landau quasiparticles and consider the system as a Fermi liquid with a fluctuating density of states. Then, (1), after taking into account (2) in the manner discussed above, can be transformed to the form:

$$
\begin{aligned}
& \frac{1}{v_{0} g}=\left\langle\int _ { 0 } ^ { \epsilon _ { p } } \frac { d \xi N ( \xi ) } { 2 \sqrt { \xi ^ { 2 } + \Delta ^ { 2 } } } \left(\tanh \frac{\sqrt{\xi^{2}+\Delta^{2}}+\mathbf{k v}}{2 T}\right.\right. \\
& \left.\left.+\tanh \frac{\sqrt{\xi^{2}+\Delta^{2}}-\mathbf{k v}}{2 T}\right)\right\rangle,
\end{aligned}
$$

where $\langle\cdots\rangle$ denotes averaging over the angles in the scalar product $\widehat{\mathbf{k}} \widehat{\mathbf{v}}$ and $\mathbf{k}=\widehat{\mathbf{k}} k_{F}$ and $\mathbf{v}=\widehat{\mathbf{v}} v$. Here, $N(\xi)$ is the dimensionless density of states function, and it is found from the given dispersion relation inherent to the studied anisotropic superconducting system. More precisely, it appears as the final product in the conformal transformation approach and can also include effects of the particle-hole asymmetry and an extra factor, as, for example, the spectral function defined in the Eliashberg equations. Since for the BCS model $N(\xi)=$ 1 , we take it in the form $N(\xi)=1+\rho(\xi)$ in further discussion. According to previous studies [38], the function $\rho$ can substantially change various parameters of a superconducting system. The effects are particularly pronounced if the function has one narrow fluctuation (peak) in the vicinity of the Fermi surface. Moreover, peaks of various shapes yield similar results. Therefore, at this point, we do not need to consider any particular analytical properties of the function $\rho$, treating it as a locally constant function.

In the next step, introducing the symbols $x=\xi / 2 T, D=$ $\Delta / 2 T, x_{p}=\xi_{p} / 2 T$, and $w=k_{F} v / 2 T$, (3) can be rewritten as

$$
\begin{aligned}
& \frac{1}{v_{0} g}=\left\langle\int _ { 0 } ^ { x _ { p } } \frac { d x [ 1 + \varrho ( x ) ] } { 2 \sqrt { x ^ { 2 } + D ^ { 2 } } } \left[\tanh \left(\sqrt{x^{2}+D^{2}}+w \widehat{\mathbf{k}} \widehat{\mathbf{v}}\right)\right.\right. \\
& \left.\left.\quad+\tanh \left(\sqrt{x^{2}+D^{2}}-w \widehat{\mathbf{k}} \widehat{\mathbf{v}}\right)\right]\right\rangle,
\end{aligned}
$$

where the function $\rho(\xi)$ is redefined into $\varrho(x)$, which can be again modeled in various manners. The factor $\left(\nu_{0} g\right)^{-1}$ can be eliminated by employing (4) in the limit cases $T=T_{c}$ and $D=0$.

In order to obtain the full representation of the gap equation of the Abrikosov-Maki type when $w=0$, we note that

$$
\begin{aligned}
\frac{\tanh \sqrt{x^{2}+D^{2}}}{\sqrt{x^{2}+D^{2}}}= & \frac{\tanh x}{x} \\
& +\sum_{l=1}^{\infty} \frac{1}{l ! 2^{l}}\left(\frac{1}{x} \frac{d}{d x}\right)^{l} \frac{\tanh x}{x} D^{2 l},
\end{aligned}
$$

where the differential operator should be read as

$$
\left(\frac{1}{x} \frac{d}{d x}\right)^{l}=\underbrace{\frac{1}{x} \frac{d}{d x} \cdots \frac{1}{x} \frac{d}{d x}}_{l} .
$$

Hence, for further calculation, we define the integrals

$$
H_{l}=\int_{0}^{\infty}\left(\frac{1}{x} \frac{d}{d x}\right)^{l} \frac{\tanh x}{x} d x,
$$

where for $l \geq 1$ we can replace $x_{p}$ by $\infty$. Substituting $x=s y$, differentiating by $s$, and putting $s=1$, we find that

$$
H_{l}=\frac{1}{2 l} \int_{0}^{\infty}\left(\frac{1}{x} \frac{d}{d x}\right)^{l} \cosh ^{-2} x d x .
$$

Let us now define an extended form of $H_{l}$ which will become useful in our discussion later on:

$$
G_{l}(n)=\int_{0}^{\infty}\left(\frac{1}{x} \frac{d}{d x}\right)^{l} \cosh ^{-2 n} x d x
$$

so $G_{l}(1)=2 l H_{l}$. After applying the differential operator in the integral $G_{l}(n)$ once, then substituting $x=s y$, differentiating by $s$, putting $s=1$, and completing some algebra, we arrive at the following recursive formula:

$$
(l-1) G_{l}(n)=2 n^{2} G_{l-1}(n)-n(2 n+1) G_{l-1}(n+1) .
$$

Note that substituting $l=1$ into the obtained formula (10), we get

$$
G_{0}(n+1)=\frac{2 n}{2 n+1} G_{0}(n),
$$

and taking into account that $G_{0}(1)=1$, we find

$$
G_{0}(n)=\frac{2^{n-1}(n-1) !}{(2 n-1) ! !} .
$$

In order to calculate $G_{1}(n)$, after integrating by parts, it can be transformed into the form:

$$
G_{1}(n)=\int_{0}^{\infty} \ln x \frac{d^{2}}{d x^{2}} \cosh ^{-2 n} x d x,
$$

from which in turn, after some algebra, we obtain

$$
G_{1}(n)=2 n(2 n+1) I_{n+1}-4 n^{2} I_{n}
$$


Here, the symbol $I_{n}$ denotes the integral

$$
I_{n}=\int_{0}^{\infty} \ln x \cosh ^{-2 n} x d x
$$

calculated in [39] and reported in the appendix.

Eventually, employing the relation (A.19), formula (14) reduces to the form:

$$
G_{1}(n)=\frac{4^{n}}{(2 n-1) !}\left[n^{2} S(n)-S(n+1)\right],
$$

from which it is easy to find

$$
\begin{aligned}
G_{1}(1)= & -14 \frac{\zeta(3)}{\pi^{2}}, \\
G_{1}(2)= & -\frac{28}{3} \frac{\zeta(3)}{\pi^{2}}-124 \frac{\zeta(5)}{\pi^{4}}, \\
G_{1}(3)= & -\frac{112}{15} \frac{\zeta(3)}{\pi^{2}}-124 \frac{\zeta(5)}{\pi^{4}}-762 \frac{\zeta(7)}{\pi^{6}}, \\
G_{1}(4)= & -\frac{32}{5} \frac{\zeta(3)}{\pi^{2}}-\frac{1736}{15} \frac{\zeta(5)}{\pi^{4}}-1016 \frac{\zeta(7)}{\pi^{6}} \\
& -4088 \frac{\zeta(9)}{\pi^{8}} .
\end{aligned}
$$

The obtained formulas (10), (16), and (A.20) allow us to evaluate $G_{l}(n)$, for $l \geq 1$, and eventually find

$$
G_{l}(n)=\frac{2^{l-1}}{(l-1) !} \frac{4^{n}}{(2 n-1) !} \sum_{j=0}^{l}(-1)^{j} B_{j}(l, n) S(n+j),
$$

where

$$
B_{j}(l, n)=\sum_{n \leq i_{1} \leq i_{2} \cdots \leq i_{n-j-1} \leq n+j} i_{1}^{2} i_{2}^{2} \cdots i_{l-j}^{2},
$$

for $j=0,1, \ldots, l-1$ and $B_{l}(l, n)=1$. Hence, in particular, for $l=2$, we have

$$
\begin{aligned}
& G_{2}(n)=\frac{2 \cdot 4^{n}}{(2 n-1) !}\left\{n^{4} S(n)\right. \\
&\left.-\left[n^{2}+(n+1)^{2}\right] S(n+1)+S(n+2)\right\},
\end{aligned}
$$

and, consistently, for $n=1,2$, and 3, their explicit forms read

$$
\begin{aligned}
& G_{2}(1)=372 \frac{\zeta(5)}{\pi^{4}}, \\
& G_{2}(2)=248 \frac{\zeta(5)}{\pi^{4}}+7620 \frac{\zeta(7)}{\pi^{6}}, \\
& G_{2}(3)=\frac{992}{5} \frac{\zeta(5)}{\pi^{4}}+7620 \frac{\zeta(7)}{\pi^{6}}+85848 \frac{\zeta(9)}{\pi^{8}} .
\end{aligned}
$$

Furthermore, for $l=3$,

$$
\begin{aligned}
& G_{3}(n)=\frac{2 \cdot 4^{n}}{(2 n-1) !}\left\{n^{6} S(n)\right. \\
& \quad-\left[n^{4}+n^{2}(n+1)^{2}+(n+1)^{4}\right] S(n+1) \\
& \left.\quad+\left[n^{2}+(n+1)^{2}+(n+2)^{2}\right] S(n+2)-S(n+3)\right\},
\end{aligned}
$$

which for $n=1$ and 2 yields

$$
\begin{aligned}
& G_{3}(1)=-11430 \frac{\zeta(7)}{\pi^{6}}, \\
& G_{3}(2)=-7620 \frac{\zeta(7)}{\pi^{6}}-429240 \frac{\zeta(9)}{\pi^{8}} .
\end{aligned}
$$

Note also that $S(m)$ can be written in the form:

$$
S(m)=[(m-1) !]^{2} I_{1}-\frac{(2 m-1) !}{4^{m-1}} I_{m},
$$

and hence the integrals $G_{l}(n)$ are expressed by means of a linear combination of $I_{m}$, where $n \leq m \leq n+l$, and $I_{1}$.

Since for the formulated problem, we have to find the integrals $H_{l}$ and $H_{l}=(1 / 2 l) G_{l}(1)$; below we focus on $G_{l}(1)$, solely. Employing (18), (A.10), and (A.19), after some algebra, we find

$$
G_{l}(1)=(-1)^{l} \frac{(2 l) !}{(l-1) !} \frac{\left(2^{2 l+1}-1\right)}{2^{l-1}} \frac{\zeta(2 l+1)}{\pi^{2 l}},
$$

and hence

$$
H_{l}=(-1)^{l}(2 l-1) ! !\left(2^{2 l+1}-1\right) \frac{\zeta(2 l+1)}{\pi^{2 l}} .
$$

In particular,

$$
\begin{aligned}
& H_{1}=-7 \frac{\zeta(3)}{\pi^{2}}, \\
& H_{2}=93 \frac{\zeta(5)}{\pi^{4}}, \\
& H_{3}=-1905 \frac{\zeta(7)}{\pi^{6}}, \\
& H_{4}=53655 \frac{\zeta(9)}{\pi^{8}} .
\end{aligned}
$$

Now, restricting the discussion to the case of $\varrho(x) \equiv 0$, we can rewrite (4) in the form (cf. [14]):

$$
\begin{aligned}
& \ln \frac{T}{T_{c}} \\
& \quad=\sum_{l=1}^{\infty}(-1)^{l} \frac{(2 l-1) ! !}{2^{l} l !}\left(2-2^{-2 l}\right) \frac{\zeta(2 l+1)}{\pi^{2 l}}\left(\frac{\Delta}{T}\right)^{2 l},
\end{aligned}
$$

where we include that, for $T=T_{c}$, the gap function $\Delta=0$ and we also assume that $\tanh \left(\xi_{p} / 2 T\right)$ can be replaced by 1 in this limit. The obtained equation allows one to find the superconducting gap $\Delta$ as a function of the temperature $T$ using the method of successive approximations. So, neglecting all terms with $l \geq 2$ in the sum, one gets the solution of the first boundary value problem for (28). Taking it as the first approximation and including the next terms in (28), one can obtain solutions in the subsequent approximations. For example, in the third step, we find

$$
\begin{aligned}
\Delta(T)= & 3.063 T_{c}\left(1-\frac{T}{T_{c}}\right)^{1 / 2}-1.254 T_{c}\left(1-\frac{T}{T_{c}}\right)^{3 / 2} \\
& +1.454 T_{c}\left(1-\frac{T}{T_{c}}\right)^{5 / 2},
\end{aligned}
$$


where it is always taken into account that $T$ is close to $T_{c}$ and should be expressed as $T=T_{c}\left[1-\left(1-T / T_{c}\right)\right]$. Moreover, using relations established in [40], we can derive the reduced specific heat difference in the form:

$$
\begin{aligned}
\frac{\Delta C(T)}{C_{N}\left(T_{c}\right)}= & 1.426-2.802\left(1-\frac{T}{T_{c}}\right) \\
& +5.948\left(1-\frac{T}{T_{c}}\right)^{2} .
\end{aligned}
$$

This allows us to find that, at $T=T_{c}$, the reduced specific heat jump is equal to 1.462 , whereas the slope of the tangent line to the reduced specific heat difference curve is

$$
\left[\frac{d}{d\left(T / T_{c}\right)} \frac{\Delta C(T)}{C_{N}\left(T_{c}\right)}\right]_{T=T_{c}}=\tan 1.228
$$

which corresponds to the angle of $70.4^{\circ}$.

Note that the sum (28) is an oscillating power series, whose coefficients for $l \rightarrow \infty$ tend to 0 as $\pi^{-2 l}$. Thus, the expression (28) converges if $T>\Delta / \pi$, and then the resulting sequence converges, yielding a solution to the problem.

2.2. Integrals of the Fundamental Equations for $\mathbf{v} \neq 0$. In developed models of superconductors, some additional parameters, such as a supercurrent or an external magnetic field, are included. In that case, one should also consider other expressions describing the superconducting current and the current of normal particles, the free energy difference, the entropy, and the magnetic susceptibility. These expressions allow one to derive a number of quantitative thermodynamic characteristics of the system such as the transition temperature, the isotope shift, the specific heat jump, the critical current, the critical superflow, the London penetration depth, the critical magnetic field, and other quantities important in characterization of superconducting systems $[7,11,20]$. Note that the integrand in the gap equation (4)-as well as in other expressions mentioned above-can be expanded in a double series, with respect to $D^{2}$ and the additional parameter $(w \widehat{\mathbf{k}} \widehat{\mathbf{v}})^{2}$. For the integrand in the gap equation, it takes the form:

$$
\begin{aligned}
& \frac{\tanh \left(\sqrt{x^{2}+D^{2}}+w \widehat{\mathbf{k}} \widehat{\mathbf{v}}\right)+\tanh \left(\sqrt{x^{2}+D^{2}}-w \widehat{\mathbf{k}} \widehat{\mathbf{v}}\right)}{2 \sqrt{x^{2}+D^{2}}} \\
& =\frac{\tanh x}{x}+\sum_{l=1}^{\infty} \frac{1}{l ! 2^{l}}\left(\frac{1}{x} \frac{d}{d x}\right)^{l} \frac{\tanh x}{x} D^{2 l}+\sum_{l=1}^{\infty} \frac{1}{(2 l) !} \\
& \quad \sum_{k=0}^{l-1}\left(\begin{array}{c}
2 l \\
2 k+2
\end{array}\right) \frac{1}{2^{2(l-k-1)}}\left(\frac{1}{x} \frac{d}{d x}\right)^{2(l-k-1)} \frac{d^{2 k+1}}{d x^{2 k+1}} \\
& \cdot \tanh x D^{4(l-k-1)}(w \widehat{\mathbf{k}} \widehat{\mathbf{v}})^{2(k+1)}+\sum_{l=1}^{\infty} \frac{1}{(2 l+1) !}
\end{aligned}
$$

$$
\begin{aligned}
& \cdot \sum_{k=0}^{l-1}\left(\begin{array}{c}
2 l+1 \\
2 k+2
\end{array}\right) \frac{1}{2^{2(l-k)-1}}\left(\frac{1}{x} \frac{d}{d x}\right)^{2(l-k)-1} \frac{d^{2 k+1}}{d x^{2 k+1}} \\
& \cdot \tanh x D^{2(2 l-2 k-1)}(w \widehat{\mathbf{k}} \widehat{\mathbf{v}})^{2(k+1)} .
\end{aligned}
$$

Hence, there appear some new specific integrals, which can be represented as

$$
L_{l}(k)=\int_{0}^{\infty}\left(\frac{1}{x} \frac{d}{d x}\right)^{l} \frac{d^{2 k+1}}{d x^{2 k+1}} \tanh x d x
$$

and rewritten in the equivalent form

$$
L_{l}(k)=\int_{0}^{\infty}\left(\frac{1}{x} \frac{d}{d x}\right)^{l} \frac{d^{2 k}}{d x^{2 k}} \cosh ^{-2} x d x .
$$

However, in order to evaluate such integrals, we yet define their extended forms:

$$
M_{l}(k, n)=\int_{0}^{\infty}\left(\frac{1}{x} \frac{d}{d x}\right)^{l} \frac{d^{2 k}}{d x^{2 k}} \cosh ^{-2 n} x d x,
$$

so that $L_{l}(k)=M_{l}(k, 1)$. Now, use the following relations for even-order derivatives of $\cosh ^{-2 n} x$, that is,

$$
\begin{aligned}
& \frac{d^{2 k}}{d x^{2 k}} \cosh ^{-2 n} x \\
& \quad=\sum_{j=0}^{k}(-1)^{j} 2^{2(k-j)} C_{j}(k, n) D(j, n) \cosh ^{-2(n+j)} x,
\end{aligned}
$$

where

$$
C_{j}(k, n)=\sum_{n \leq i_{1} \leq i_{2} \cdots \leq i_{k-j} \leq n+j} i_{1}^{2} i_{2}^{2} \cdots i_{k-j}^{2},
$$

for $j=0,1, \ldots, k-1$ and $C_{k}(k, n)=1$, and

$$
D(j, n)=\prod_{i=0}^{j-1} 2(n+i)[2(n+i)+1]
$$

for $j=1,2, \ldots, k$ and $D(0, n)=1$. Note that $C_{0}(k, n)=n^{2 k}$, and

$$
\begin{aligned}
& C_{k-1}(k, n)=\frac{1}{6}[(n+k-1)(n+k)(2 n+2 k-1) \\
& -(n-1) n(2 n-1)],
\end{aligned}
$$

and including (9), we eventually find that

$$
\begin{aligned}
& M_{l}(k, n) \\
& \quad=\sum_{j=0}^{k}(-1)^{j} 2^{2(k-j)} C_{j}(k, n) D(j, n) G_{l}(n+j),
\end{aligned}
$$


and hence putting $n=1$, we obtain

$$
L_{l}(k)=\sum_{j=0}^{k}(-1)^{j} 2^{2(k-j)} C_{j}(k, 1) D(j, 1) G_{l}(1+j),
$$

where the integrals $G_{l}(n)$ are expressed by means of formula (18), and, for example,

$$
\begin{aligned}
& L_{1}(2)=48 H_{3}, \\
& L_{2}(1)=24 H_{3}, \\
& L_{l}(0)=G_{l}(1)=2 l H_{l},
\end{aligned}
$$

for $l \geq 1$ and $L_{0}(0)=G_{0}(1)$. Note also that

$$
\left\langle(\widehat{\mathbf{k}} \widehat{\mathbf{v}})^{2 p}\right\rangle= \begin{cases}\frac{1}{2^{2 p}}\left(\begin{array}{c}
2 p \\
p
\end{array}\right) & \text { for 2D systems } \\
\frac{1}{2 p+1} & \text { for 3D systems }\end{cases}
$$

and such averages vanish for odd exponents.

\section{Conclusions}

In the present paper, we have found and discussed some infinite families of specific integrals inherent in models of superconducting systems. The results presented here can be easily applied in analytical studies on superconductors of the new generation, since the gap equation and the carrier concentration equation along with the other particular equations mentioned in the paper are important tools in research of thermodynamic properties of superconductors. They are commonly used to derive fundamental quantitative characteristics for various classes of superconducting systems.

In order to apply the results presented in the paper to superconductors of the new generation studied within the conformal transformation method [7], it is enough to take into account a model form of the function $\varrho(x)$ - which so far has been treated as constant -in a local interval around $x=0$, where the integrands in the formulas under discussion are strongly concentrated. The model function should possess a narrow fluctuation (peak) in the vicinity of the Fermi surface. Since various particular shapes of the fluctuation produce similar results, we propose to take the function $\varrho(x)$ in the following normalized form:

$$
\varrho(x)=\chi \frac{(2 p-1) ! !}{2^{p-1}(p-1) !} \cosh ^{-2 p} x,
$$

where we also include (12). The fixed parameter $\chi$ is the height of the local fluctuation, whereas $\eta=2 \operatorname{arccosh}(\sqrt[2 n]{2})$ denotes its half-width. These should be estimated in accordance with a given form of the dispersion relation $\epsilon_{\mathbf{k}}(\mathrm{cf}$. [7, 38]). Subsequently, the form of fluctuation $[1+\varrho(x)]$ can be substituted into all integrals $G_{l}(n)$ and $M_{l}(k, n)$. Because of the function $\varrho(x) \propto \cosh ^{-2 p} x$ there appear some extra integrals $G_{l}(n+p)$ and $M_{l}(k, n+p)$ in the equations under consideration. In such a way, the proposed form of $\varrho(x)$ allows us to employ the derived integrals to calculate thermodynamic parameters of composed superconductors [3, 4, 6, 8-10, 12, 14, 21].

Let us also emphasize that although the case $w=0$ corresponds to the Van Hove Scenario, the case $w>0$ can be properly discussed in the conformal transformation method only $[7,21-23,30]$. One should then include additional functions $\varrho_{2 i}(x)$, for $i=1,2, \ldots$, where $\varrho_{0}(x) \equiv \varrho(x)$, which appear after averaging the dimensionless kernel of the density of states with $(\widehat{\mathbf{k}} \widehat{\mathbf{v}})^{2 i}$ over the angles and can be modeled similarly to $\varrho(x)$.

\section{Appendix}

\section{The Integral $I_{n}$}

As we have shown in [39], the formula for the family of integrals

$$
I_{n}=\int_{0}^{\infty} \ln x \cosh ^{-2 n} x d x,
$$

for $n \in \mathcal{N}$, can be derived by analytical calculations. In order to calculate $I_{n}$, we introduce the integrals:

$$
J_{n}(s)=\int_{0}^{\infty} f_{n}(s, u) d u,
$$

where

$$
f_{n}(s, u)=u^{s} \cosh ^{-2 n} u
$$

and $s$ is a complex number such that $\operatorname{Re} s>-1$. Then, for each $n \in \mathcal{N}$, the function $f_{n}(s, u)$ is a continuous function of $s$ and $u$, where $s$ ranges over the region $\mathscr{D}=\{s: \operatorname{Re} s>-1\}$ and $u \in[a, A]$, for all $0<a<A<\infty$. Moreover, for every value of $u \in[a, A]$, the function $f_{n}(s, u)$ is an analytic function of $s \in \mathscr{D}$, and finally the integral $J_{n}(s)$, for all $n \in \mathcal{N}$, is almost uniformly convergent in the domain $\mathscr{D}$. Therefore, $J_{n}(s)$ is an analytic function of $s \in \mathscr{D}$ and one can differentiate under the integral sign [41]:

$$
J_{n}^{\prime}(s)=\int_{0}^{\infty} \frac{\partial f_{n}(s, u)}{\partial s} d u
$$

In particular, it yields

$$
I_{n}=J_{n}^{\prime}(0)
$$

Let us first derive a formula for $J_{n}(s)$. Using a series expansion of $f_{n}(s, u)$, for $u>0$, we obtain

$$
f_{n}(s, u)=4^{n} \sum_{k=0}^{\infty}(-1)^{k}\left(\begin{array}{c}
2 n+k-1 \\
k
\end{array}\right) u^{s} e^{-2 u(n+k)},
$$

which, after changing the summation index $k$ to $m=n+k$ and using the formula

$$
\begin{aligned}
& \frac{(n+m-1) !}{(m-n) !} \\
& \quad=\left(m^{2}-(n-1)^{2}\right)\left(m^{2}-(n-2)^{2}\right) \cdots\left(m^{2}-1\right) m,
\end{aligned}
$$


transforms into

$$
\begin{aligned}
f_{n}(s, u)= & \frac{4^{n}}{(2 n-1) !} \sum_{k=0}^{\infty}(-1)^{m+n}\left(m^{2}-(n-1)^{2}\right) \\
& \cdot\left(m^{2}-(n-2)^{2}\right) \cdots\left(m^{2}-1\right) m u^{s} e^{-2 u m},
\end{aligned}
$$

where the summation can be extended to $m$ running from 1 to $\infty$ since for $m=1, \ldots, n-1$ we obtain zero terms. If $\operatorname{Re} s$ is sufficiently large, we can integrate the series term-by-term to get

$$
\begin{aligned}
& J_{n}(s)=\frac{4^{n}}{(2 n-1) !} \sum_{k=0}^{\infty}(-1)^{m+n}\left(m^{2}-(n-1)^{2}\right) \\
& \cdot\left(m^{2}-(n-2)^{2}\right) \cdots\left(m^{2}-1\right) m \int_{0}^{\infty} u^{s} e^{-2 u m} d u \\
& =\frac{4^{n}}{(2 n-1) !} \sum_{k=0}^{\infty}(-1)^{m+n}\left(m^{2}-(n-1)^{2}\right) \\
& \cdot\left(m^{2}-(n-2)^{2}\right) \cdots\left(m^{2}-1\right) \frac{1}{(2 m)^{s}} \Gamma(s+1),
\end{aligned}
$$

where the change of variables $2 u m=x$ in the integrals has been performed.

The precise argument which allows term-by-term integration goes as follows. First, we note that term-by-term integration of the series (A.8) is justified for the integration from $a$ to $A$, where $0<a<A<\infty$ since the series (A.8) is uniformly convergent for $u \in[a, A]$ and any $s \in$ $\mathscr{D}$. This allows us to integrate (A.8) term-by-term also over the interval $(0, \infty)$ provided the right-hand side of (A.9) converges absolutely [41]. It is the case in the half-plane $\operatorname{Re} s>$ $2 n-1$, and hence in that half-plane the formula (A.9) is valid.

In order to find the coefficients standing by the same power of $m$ in (A.9), let us introduce

$$
A_{j}(n)=\sum_{1 \leq i_{1}<i_{2} \cdots<i_{n-j-1} \leq n-1} i_{1}^{2} i_{2}^{2} \cdots i_{n-j-1}^{2}
$$

for $j=0,1, \ldots, n-2$ and $A_{n-1}(n)=1$. Thus, in particular,

$$
\begin{aligned}
A_{0}(n) & =[(n-1) !]^{2}, \\
A_{n-2}(n) & =\frac{1}{6}(n-1) n(2 n-1) .
\end{aligned}
$$

For example,

$$
\begin{aligned}
& A_{1}(3)=3, \\
& A_{1}(4)=49, \\
& A_{2}(4)=14, \\
& A_{1}(5)=820, \\
& A_{2}(5)=273,
\end{aligned}
$$

$$
\begin{aligned}
& A_{3}(5)=30, \\
& A_{1}(6)=21076, \\
& A_{2}(6)=7645, \\
& A_{3}(6)=1023, \\
& A_{4}(6)=55 .
\end{aligned}
$$

Now using (A.10) and the formula

$$
\begin{aligned}
& \sum_{m=0}^{\infty}(-1)^{m-1} m^{-(s-2 j)} \\
& \quad=\left(1-2^{1+2 j-s}\right) \zeta(s-2 j) 2^{-s} \Gamma(s+1),
\end{aligned}
$$

for $\operatorname{Re} s>2 n-2$, where $\zeta$ is the Riemann $\zeta$-function, we arrive at

$$
\begin{aligned}
J_{n}(s)= & \frac{4^{n}}{2(2 n-1) !} \sum_{j=0}^{\infty}(-1)^{j} A_{j}(n)\left(1-2^{1+2 j-s}\right) \\
& \cdot \zeta(s-2 j) 2^{-s} \Gamma(s+1) .
\end{aligned}
$$

By analytic continuation, this formula holds for all $s \in \mathscr{D}$ and in fact yields the analytic continuation of $J_{n}(s)$ even to the left of the half-plane $\operatorname{Re} s>-1$ with poles at $s=-1,-2,-3, \ldots$.

Recall that $\zeta(-2 j)=0$, for $j=1,2, \ldots, \zeta(0)=-1 / 2$, and $\Gamma(1)=1$. Differentiating (A.14) and then putting $s=0$, we obtain

$$
\begin{aligned}
I_{n} & =\frac{4^{n}[(n-1) !]^{2}}{(2 n-1) !}\left[\frac{d}{d s} 2^{-s}\left(1-2^{1-s}\right) \Gamma(s+1)\right. \\
& \cdot \zeta(s)]_{s=0}+\frac{4^{n}}{2(2 n-1) !} \sum_{j=1}^{n-1}(-1)^{j} A_{j}(n)(1 \\
& \left.-2^{1+2 j}\right) \zeta^{\prime}(-2 j) .
\end{aligned}
$$

Next, using the Riemann functional equation [31, 42]

$$
\Gamma\left(\frac{s}{2}\right) \zeta(s)=\pi^{(1 / 2)(s-1)} \Gamma\left(\frac{1-s}{2}\right) \zeta(1-s),
$$

we arrive at the formula

$$
\zeta^{\prime}(-2 j)=\frac{(-1)^{j}(2 j) !}{2(2 \pi)^{2 j}} \zeta(2 j+1) .
$$

Now, using formula (A.17) and formulas

$$
\begin{aligned}
& \Gamma^{\prime}(1)=-C, \\
& \zeta^{\prime}(0)=-\frac{1}{2} \ln (2 \pi),
\end{aligned}
$$

where $C$ is the Euler constant $(C=0.577215 \ldots)$, we finally obtain

$$
\begin{aligned}
I_{n} & =-\frac{4^{n-1}[(n-1) !]^{2}}{(2 n-1) !} \ln \frac{4 e^{C}}{\pi}-\frac{4^{n-1}}{(2 n-1) !} S(n), \\
S(n) & =\sum_{j=1}^{n-1} A_{j}(n) \frac{\left(2^{2 j+1}-1\right)(2 j) !}{(2 \pi)^{2 j}} \zeta(2 j+1),
\end{aligned}
$$


with $S(1)=0$. Consistently, we can find $I_{n}$ in particular cases, and for $n=1$ we have

$$
I_{1}=-\ln \frac{4 e^{C}}{\pi},
$$

in agreement with other results [3-5, 12, 14, 15, 31, 39, 43, 44]. Moreover, for subsequent $n$ 's, we obtain

$$
\begin{aligned}
& I_{2}=\frac{2}{3} I_{1}-\frac{2}{3} S(2), \\
& I_{3}=\frac{8}{15} I_{1}-\frac{2}{15} S(3), \\
& I_{4}=\frac{16}{35} I_{1}-\frac{4}{315} S(4), \\
& I_{5}=\frac{128}{315} I_{1}-\frac{2}{2835} S(5),
\end{aligned}
$$

where

$$
\begin{aligned}
S(2) & =\frac{7}{2} \frac{\zeta(3)}{\pi^{2}}, \\
S(3) & =\frac{1}{2}\left[35 \frac{\zeta(3)}{\pi^{2}}+93 \frac{\zeta(5)}{\pi^{4}}\right], \\
S(4) & =\frac{1}{4}\left[686 \frac{\zeta(3)}{\pi^{2}}+2604 \frac{\zeta(5)}{\pi^{4}}+5715 \frac{\zeta(7)}{\pi^{6}}\right], \\
S(5) & =\frac{1}{2}\left[5740 \frac{\zeta(3)}{\pi^{2}}+25389 \frac{\zeta(5)}{\pi^{4}}+85725 \frac{\zeta(7)}{\pi^{6}}\right. \\
+ & \left.160965 \frac{\zeta(9)}{\pi^{8}}\right] .
\end{aligned}
$$

Moreover,

$$
\begin{aligned}
& \zeta(3)=1.2020569032, \\
& \zeta(5)=1.0369277551, \\
& \zeta(7)=1.0083492774, \\
& \zeta(9)=1.0020083928,
\end{aligned}
$$

and $\zeta(x) \rightarrow 1$, for $x \rightarrow \infty$, which can be exploited in numerical estimations.

\section{Conflict of Interests}

The authors declare that there is no conflict of interests regarding the publication of this paper.

\section{Acknowledgment}

This study is supported by MNiSW in 2015/2016.

\section{References}

[1] Y. Kamihara, H. Hiramatsu, M. Hirano et al., "Iron-based layered superconductor: LaOFeP," Journal of the American Chemical Society, vol. 128, no. 31, pp. 10012-10013, 2006.
[2] Y. Kamihara, T. Watanabe, M. Hirano, and H. Hosono, "Ironbased layered superconductor $\mathrm{La}\left[\mathrm{O}_{1-x} \mathrm{~F}_{x}\right] \mathrm{FeAs}(x=0.05-0.12)$ with $T_{c}=26 \mathrm{~K}$," Journal of the American Chemical Society, vol. 130, no. 11, pp. 3296-3297, 2008.

[3] A. L. Fetter and J. D. Walecka, Quantum Theory of Many-Particle Systems, McGraw-Hill, 1971.

[4] A. A. Abrikosov, L. P. Gorkov, and I. E. Dzyaloshinski, Methods of Quantum Field Theory in Statistical Physics, Fizmatgiz, Moscow, Russia, 1962.

[5] A. V. Svidzynskii, Space-Inhomogeneous Problems of the Theory of Superconductivity, chapter 1, Nauka, Moscow, Russia, 1982.

[6] M. Cyrot and D. Pavuna, Introduction to Superconductivity and High-T ${ }_{c}$ Materials, chapter 7.1, World Scientific, Singapore, 1992.

[7] M. Krzyzosiak, R. Gonczarek, A. Gonczarek, and L. Jacak, "Conformal transformation method in studies of high- $T_{c}$ superconductors-beyond the van Hove Scenario," in Superconductivity and Superconducting Wires, D. Matteri and L. Futino, Eds., chapter 5, Nova Science Publishers, Hauppauge, NY, USA, 2010.

[8] R. Szczęśniak and D. Szczęśniak, "Superconductivity in the presence of a pseudogap induced by local charge density waves," Chemistry of Metals and Alloys, vol. 4, pp. 50-57, 2011.

[9] R. Szczęśniak and A. P. Durajski, “The characterization of highpressure superconducting state in $\mathrm{Si}_{2} \mathrm{H}_{6}$ compound: the strongcoupling description," Journal of Physics and Chemistry of Solids, vol. 74, pp. 641-646, 2013.

[10] R. Szczęśniak and A. P. Durajski, "On the critical temperature and the energy gap in dense $\mathrm{SiH}_{4}\left(\mathrm{H}_{2}\right)_{2}$ at $250 \mathrm{GPa}$," Solid State Communications, vol. 153, no. 1, pp. 26-30, 2013.

[11] R. Gonczarek and M. Krzyzosiak, "Model of superconductivity in the singular fermi liquid," in Progress in Superconductivity Research, O. A. Chang, Ed., chapter 6, Nova Science Publishers, Hauppauge, NY, USA, 2008.

[12] K. Maki, "Effect of Pauli Paramagnetism on Magnetic Properties of High-Field Superconductors," Physical Review, vol. 148, no. 1, pp. 362-369, 1966.

[13] A. A. Abrikosov, "On the magnetic properties of superconductors of the second group," Zhurnal Eksperimentalnoi i Teoreticheskoi Fiziki, vol. 32, p. 1442, 1957 (Russian), Reprinted in: Journal of Experimental and Theoretical Physics, vol. 5, p. 1174, 1957.

[14] A. A. Abrikosov, Fundamentals of the Theory of Metals, Nauka, Moscow, Russia, 1962.

[15] D. Kasinathan, K.-W. Lee, and W. E. Pickett, "On heavy carbon doping of $\mathrm{MgB}_{2}$," Physica C: Superconductivity and Its Applications, vol. 424, no. 3-4, pp. 116-124, 2005.

[16] J. Kortus, O. V. Dolgov, R. K. Kremer, and A. A. Golubov, "Band filling and interband scattering effects in $\mathrm{MgB}_{2}$ : carbon versus aluminum doping," Physical Review Letters, vol. 94, Article ID 027002, 2005.

[17] W. S. Agrestini, C. Metallo, M. Filippi et al., "Substitution of Sc for $\mathrm{Mg}$ in $\mathrm{MgB}_{2}$ : effects on transition temperature and Kohn anomaly," Physical Review B, vol. 70, no. 13, Article ID 134514, 2004.

[18] H. Mori, T. Okano, M. Kamiya et al., "Bandwidth and band filling control in organic conductors," Physica C: Superconductivity, vol. 357-360, no. 2, pp. 103-107, 2001.

[19] R. Gonczarek, M. Krzyzosiak, and A. Gonczarek, "Islands of stability of the d-wave order parameter in s-wave anisotropic superconductors," European Physical Journal B, vol. 61, no. 3, pp. 299-308, 2008. 
[20] R. Gonczarek and M. Krzyzosiak, "Conformal transformation method and symmetry aspects of the group $\mathrm{C}_{4 \mathrm{v}}$ in a model of high- $\mathrm{T}_{\mathrm{c}}$ superconductors with anisotropic gap," Physica $C$, vol. 426, no. 1, pp. 278-282, 2005.

[21] R. Gonczarek, L. Jacak, M. Krzyzosiak, and A. Gonczarek, "Competition mechanism between singlet and triplet superconductivity in the tight-binding model with anisotropic attractive potential," European Physical Journal B, vol. 49, no. 2, pp. 171186, 2006.

[22] R. Gonczarek, M. Krzyzosiak, L. Jacak, and A. Gonczarek, "Coexistence of spin-singlet s- and d-wave and spin-triplet pwave order parameters in anisotropic superconductors," Physica Status Solidi B: Basic Research, vol. 244, no. 10, pp. 3559-3571, 2007.

[23] R. Gonczarek, M. Gladysiewicz, and M. Mulak, "On posibble formalism of anisoropic Fermi liqued and BCS-TYPE superconductivity," International Journal of Modern Physics B, vol. 15, no. 5, p. 491, 2001.

[24] F. C. Zhang and T. M. Rice, "Effective Hamiltonian for the superconducting $\mathrm{Cu}$ oxides," Physical Review B, vol. 37, no. 7, pp. 3759-3761, 1988.

[25] D. Y. Xing, M. Liu, Y.-G. Wang, and J. Dong, "Analytic approach to the antiferromagnetic van Hove singularity model for high$T_{c}$ superconductors," Physical Review B, vol. 60, no. 13, pp. 9775-9781, 1999.

[26] R. Szczęśniak, "Pairing mechanism for the high- $\mathrm{T}_{\mathrm{C}}$ superconductivity: symmetries and thermodynamic properties," PLoS ONE, vol. 7, no. 4, Article ID e31873, 2012.

[27] E. Pavarini, I. Dasgupta, T. Saha-Dasgupta, O. Jepsen, and O. K. Andersen, "Band-structure trend in hole-doped cuprates and correlation with $T_{c \max }$ " Physical Review Letters, vol. 87, no. 4, Article ID 047003, 2001.

[28] O. K. Andersen, A. I. Liechtenstein, O. Jepsen, and F. Paulsen, "LDA energy bands, low-energy hamiltonians, $t^{\prime}, t^{\prime \prime}, t_{\perp}(k)$, and $J_{\perp}$," Journal of Physics and Chemistry of Solids, vol. 56, pp. 15731591, 1995.

[29] O. K. Andersen, S. Y. Savrasov, O. Jepsen, and A. I. Liechtenstein, "Out-of-plane instability and electron-phonon contribution to $s$ - and $d$-wave pairing in high-temperature superconductors; LDA linear-response calculation for doped $\mathrm{CaCuO}_{2}$ and a generic tight-binding model," Journal of Low Temperature Physics, vol. 105, no. 3-4, pp. 285-304, 1996.

[30] R. Gonczarek and M. Krzyzosiak, "Some universal relations between the gap and thermodynamic functions plausible for various models of superconductors," Physica Status Solidi B: Basic Research, vol. 238, no. 1, pp. 29-39, 2003.

[31] I. S. Gradstein and I. M. Ryzhik, Tables of Integrals, Sums, Series and Products, chapter 3.73, Nauka, Moscow, Russia, 1987.

[32] R. Gonczarek, "Influence of the Fermi liquid interaction on the behaviour of superconductors in the strong magnetic fields," Acta Physica Polonica A, vol. 71, p. 59, 1987.

[33] R. Gonczarek, M. Krzyzosiak, A. Gonczarek, and L. Jacak, "New classes of integrals inherent in the mathematical structure of extended equations describing superconducting systems," International Journal of Modern Physics B, vol. 29, no. 17, Article ID 1550117, 12 pages, 2015.

[34] R. Gonczarek, "Critical current and depairing superflow in the two-dimensional superconducting Fermi system," Acta Physica Polonica A, vol. 75, p. 675, 1988.

[35] C. M. Varma, Z. Nussinov, and W. van Saarloos, "Singular or non-Fermi liquids," Physics Reports: A Review Section of Physics Letters, vol. 361, no. 5-6, pp. 267-417, 2002.
[36] R. Gonczarek, M. Gladysiewicz, and M. Mulak, "Equilibrium states and thermodynamical properties of D-wave paired HTSC in the Tight-Binding model," Physica Status Solidi (B), vol. 233, pp. 351-363, 2002.

[37] R. Gonczarek, M. Krzyzosiak, and M. Mulak, "Valuation of characteristic ratios for high- $T_{c}$ superconductors with anisotropic gap in the conformal transformation method," Journal of Physics A. Mathematical and General, vol. 37, no. 18, pp. 48994911, 2004.

[38] R. Gonczarek and M. Mulak, "Enhancement of critical temperature of superconductors implied by the local fluctuation of EDOS," Physics Letters, Section A: General, Atomic and Solid State Physics, vol. 251, no. 4, pp. 262-268, 1999.

[39] R. Gonczarek and J. Czerwonko, "On a class of integrals appearing in subcritical thermodynamics of superconductors," Bulletin de l'Académie Polonaise des Sciences. Série des Sciences Mathématiques, vol. 48, p. 403, 2000.

[40] M. Mulak and R. Gonczarek, "Discontinuous phase transitions in S-paired fermi systems," Acta Physica Polonica A, vol. 92, no. 6, pp. 1177-1189, 1997.

[41] E. C. Titchmarsh, The Theory of Functions, Oxford University Press, Oxford, UK, 1968.

[42] H. Bateman and A. Erdelyi, Eds., Higher Transcendental Functions, vol. 1, McGraw-Hill, New York, NY, USA, 1953.

[43] R. Gonczarek, "On Abrikosov's and Maki's theories of type-II superconductors in the paramagnetic green function approach," Acta Physica Polonica A, vol. 73, no. 5, pp. 683-704, 1988.

[44] J. Bardeen, L. N. Cooper, and J. R. Schrieffer, "Microscopic theory of superconductivity," Physical Review, vol. 106, no. 1, pp. 162-164, 1957. 

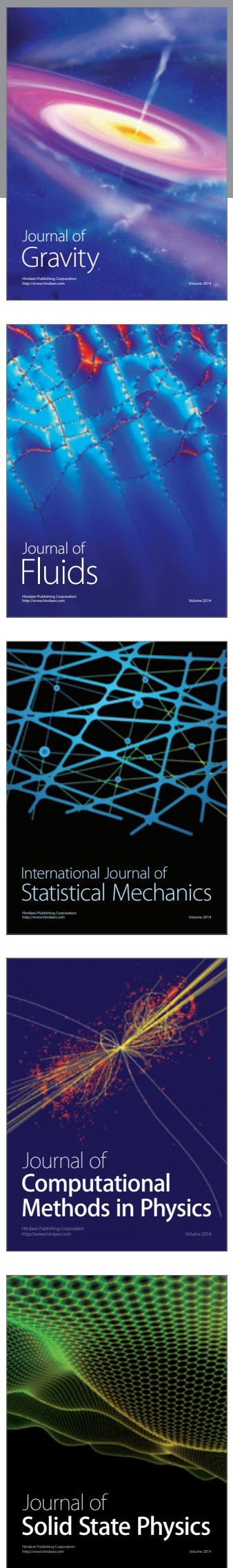

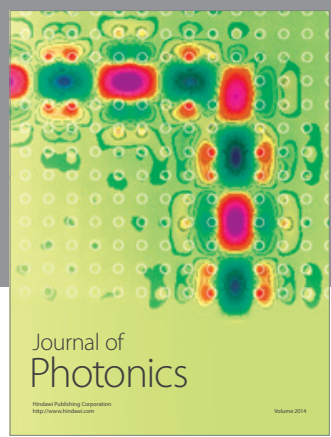

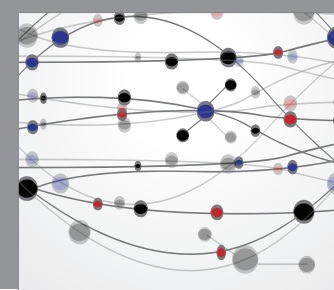

The Scientific World Journal

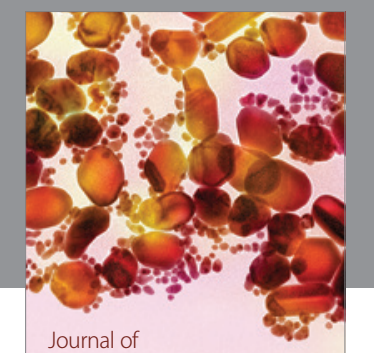

Soft Matter
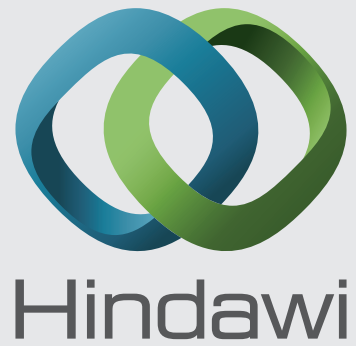

Submit your manuscripts at

http://www.hindawi.com
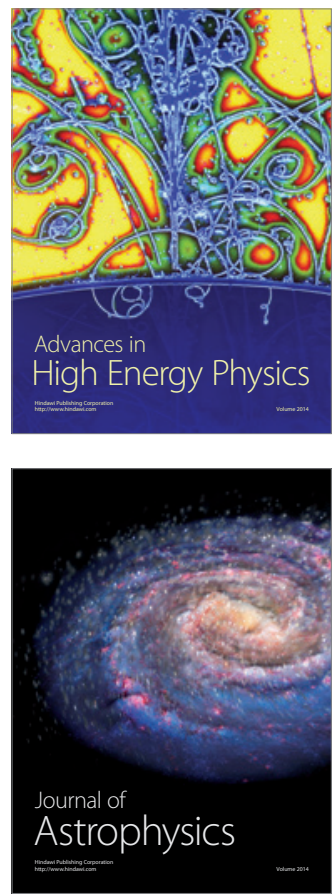
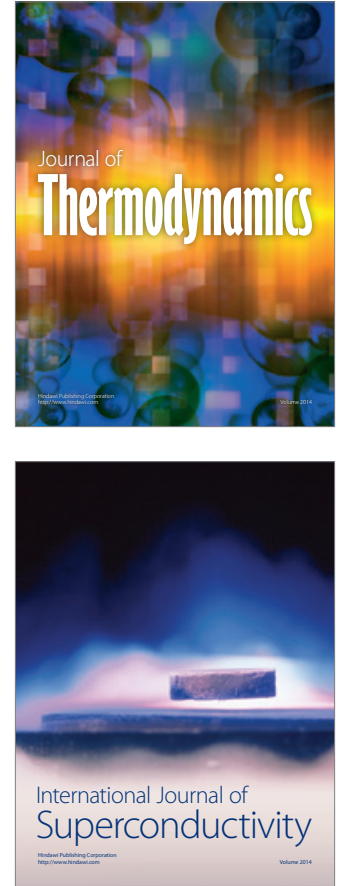
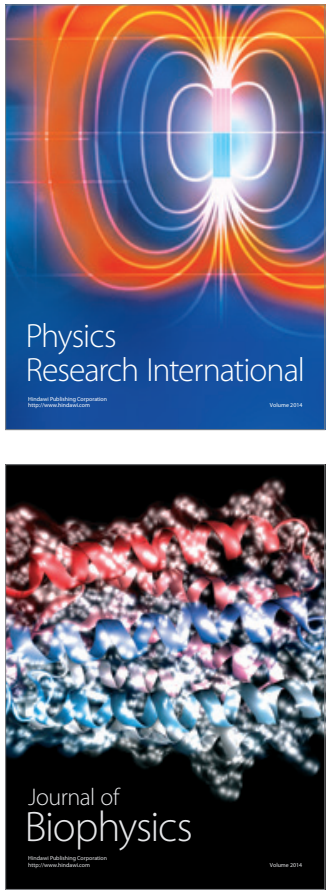
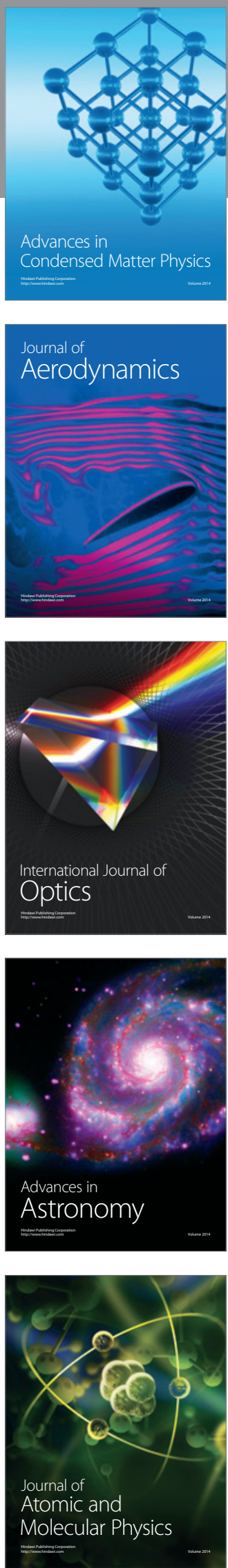